\title{
Effetti precoci e tardivi dell'eritropoietina sul metabolismo glicidico in pazienti emodializzati
}

\author{
V. Allegra, L. Martimbianco, A. Vasile \\ Servizio Emodialisi, Ospedale di Palmanova, Regione Friuli-Venezia Giulia
}

eritropoietina ricombinante umana (rHuEPO) è una terapia efficace nel trattamento dell'anemia dell'insufficienza renale cronica (1-4), ma le alte dosi necessarie per far aumentare i valori di emoglobina $(\mathrm{Hb})$ nei pazienti emodializzati (MHP) possono esplicare effetti significativi anche su organi diversi dalle cellule eritroidi. Alcuni studi hanno segnalato che il trattamento con rHuEPO nei MHP è in grado di indurre significative modificazioni del metabolismo proteico $(5,6)$ e lipidico $(7-9)$. Al contrario pochi e contrastanti lavori sono riportati in letteratura sugli effetti dell'rHuEPO sul metabolismo glicidico (10-13). Alcuni Autori hanno trovato un miglioramento della secrezione insulinica $(10,11)$ o della sensibilità all'insulina (12) in MHP dopo terapia con rHuEPO, ma questi risultati non sono stati confermati da altri (13).

È inoltre ancora controverso se gli effetti dell'rHuEPO sul metabolismo glicidico siano da attribuire alla correzione dell'anemia o ad un'azione diretta dell'rHuEPO su alcune tappe metaboliche. Recenti studi hanno dimostrato che i globuli rossi partecipano attivamente all'omeostasi glicidica (14) e che l'ipossia altera la secrezione insulinica in pancreas isolati e perfusi di cane e di ratto (15). Alcuni Autori nei MHP in terapia con rHuEPO hanno trovato una correlazione positiva tra miglioramento del metabolismo glicidico ed aumento dei valori di $\mathrm{Hb}$ (11) o della $\mathrm{pO}_{2}$ capillare (12). Al contrario altri Autori, in pazienti uremici dializzati e non dializzati, non hanno trovato alcuna correlazione tra anomalie del metabolismo glicidico e grado dell' anemia (16) e nessun effetto della correzione dell'anemia mediante emotrasfusioni sul metabolismo glicidico in MHP $(13,16)$.

Lo scopo di questo studio è di valutare gli effetti acuti e cronici della terapia con rHuEPO sul metabolismo glicidico in MHP e se questi effetti sono dovuti alla correzione dell'anemia o ad un'azione dell'rHuEPO su alcune tappe metaboliche.

\section{Materiali e metodi}

Sono stati studiati 20 pazienti ( 8 maschi e 12 femmine) di età compresa tra 21 e 70 anni $(49.6 \pm 3.2$; media \pm SE) e di età dialitica compresa tra 5 e 155 mesi (51 \pm 10$)$. Tutti presentavano anamnesi negativa per malattie epatiche ed endocrine, anamnesi familiare negativa per diabete, non presenza di diabete prima dell'inizio dell'insufficienza renale. Tutti erano esenti da malattie ematologiche. La deficienza marziale è stata esclusa con la formula di Allegra et al (17). La loro malattia primitiva era glomerulonefrite in 10 casi, nefroangiosclerosi in 3 , sindrome di Alport in uno, nefropatia ostruttiva in uno, pielonefrite in uno, nefropatia da reflusso in uno, rene policistico in uno, displasia renale in uno, sconosciuta in uno. Gli schemi di dialisi erano i seguenti: 4 h tre volte la settimana, filtro capillare o a piastra con membrana in cuprophan di 0.96-1.3 $\mathrm{m}^{2}$ di superficie e 6.5-8 $\mu \mathrm{m}$ di spessore, flusso ematico $300-350 \mathrm{ml} / \mathrm{min}$, flusso dialisato 500 $\mathrm{ml} / \mathrm{min}$. L'eparinizzazione è stata eseguita come segue: priming $1000 \mathrm{IU}$, seguita da infusione continua di $17 \mathrm{IU} / \mathrm{min}$ nella linea arteriosa. Nessun paziente assumeva farmaci quali calcio antagonisti o Bbloccanti, tutti assumevano chelanti del fosforo, calcio e calcitriolo. Un paziente in terapia antiipertensiva con captopril ha interrotto il trattamento due giomi prima di ciascun test.

Tutti i pazienti sono stati sottoposti a IVGTT prima dell'inizio della terapia. La tecnica dell'IVGTT è stata descritta in precedenti indagini $(18,19)$. In 8 pazienti l'IVGTT è stato ripetuto, la settimana successiva, 14-20h dopo una singola somministrazione endovenosa di 50 IU di rHuEPO. In 12 casi l'IVGTT è stato ripetuto dopo 3 mesi di terapia con $\mathrm{rHuEPO}$, alla dose di $50 \mathrm{IU} / \mathrm{kg}$ insieme a $20 \mathrm{mg}$ di $\mathrm{Fe}^{++}$sotto forma di Fe-gluconato-Na alla fine di ciascuna seduta dialitica e due settimane dopo una sospensione temporanea della terapia con rHuEPO. 
Tutti i test sono stati eseguiti il mattino successivo al secondo trattamento dialitico settimanale. Nel campione basale di ciascun test sono stati dosati $\mathrm{Hb}$, ematocrito $(\mathrm{Ht})$, globuli rossi (RBC) e piastrine, azoto ureico (BUN), creatinina sierica (s-Cr), acido urico (s-UA) e fosforo inorganico $(\mathrm{Pi})$ mediante autoanalyzer, eritropoietina plasmatica ( $\mathrm{pEPO}$ ) mediante dosaggio radioimmunologico. Nei due giorni precedenti ciascun test sono stati calcolati il Kt/V e il Protein Catabolic Rate (PCR) mediante la formula di Gotch e Sargent (20). È stato considerato peso corporeo secco (DBW) il più basso peso raggiunto alla fine del trattamento dialitico nella settimana precedente ciascuna valutazione.

Il glucosio plasmatico $(\mathrm{G})$ è stato dosato con il metodo della glucosio ossidasi, l'insulina immunoreattiva (IRI) e il Cpeptide (C-p) con metodo radioimmunologico (Biodata). Per ciascun test è stata calcolata la costante di utilizzazione del glucosio (K-G). G, IRI e C-p sono stati inoltre espressi come area di risposta sopra la linea di base, calcolata con il metodo dell'integrazione trapezoidale. La produzione insulinica inoltre è stata valutata in relazione all'entità della stimolazione glicidica della B-cellula pancreatica, mediante calcolo dell'indice insulinogenico (IGI; rapporto Area IRI/Area G). L'indice di insulinoresistenza (RI) è stato calcolato mediante rapporto Area IRI/K-G.

I sopramenzionati parametri, derivati dall'IVGTT, sono ritenuti degli indici abbastanza sensibili e molto utili per la valutazione del metabolismo glicidico, soprattutto in esperimenti appaiati e in studi clinici $(18,21-23)$. Il K-G è un indice molto sensibile per misurare l'utilizzazione del glucosio da parte dei tessuti periferici ed è ampiamente usato per valutare la tolleranza glicidica $(22,23)$. L'area IRI esprime il bilancio netto tra produzione e degradazione insulinica in un definito intervallo di tempo, mentre l'area C-p esprime solo il rilascio insulinico da parte delle $ß$-cellule pancreatiche. La valutazione comparata di questi due parametri è comunemente ritenuta un valido metodo per la stima della produzione insulinica globale. L'IGI misura il rilascio insulinico in relazione allo stimolo esercitato a livello della $B$-cellula pancreatica e può essere assunto come misura della sensibilità al glucosio della ß-cellula pancreatica $(18,22)$. L'RI derivato dall'IVGTT è me- no sensibile di quello derivato dall'hyperglycemic o dall'euglycemic clamp technique. Tuttavia la sua sensibilità è abbastanza elevata in esperimenti appaiati, per la bassa variabilità di risposta all'IVGTT nello stesso paziente $(18,22)$. Inoltre l'IVGTT è ben accettato dai pazienti anche in studi in cui sia necessario eseguire diversi test.

Tutti i dati sono presentati come media \pm SE. Per l'analisi statistica sono stati utilizzati il test t di Student per dati appaiati (effetti acuti), ed il test di Student-Newmann-Keuls per gruppi randomizzati (effetti cronici).

\section{Risultati}

\section{Effetti precoci}

Sedici-venti ore dopo una singola dose di rHuEPO, i livelli di pEPO sono au- mentati significativamente (da $12.2 \pm 4.5$ a $149 \pm 38.8 \mathrm{ng} / \mathrm{ml}$; $\mathrm{p}<0.01$ ), ma i valori di $\mathrm{Hb}$ non si sono modificati $(9.7 \pm 0.4$ vs $9.6 \pm 0.5 \mathrm{~g} / \mathrm{dl})$. Le curve di G e IRI (Fig. 1) e i parametri del metabolismo glicidico (Tab. I) non hanno mostrato variazioni significative.

\section{Effetti tardivi}

Dopo tre mesi di terapia con rHuEPO $11 / 12$ pazienti hanno riferito miglioramento della Koro sensazione generale di benessere, dell'attività fisica e dell'appetito. In Tabella II sono riassunti i parametri di laboratorio e gli indici dialitici prima e dopo la terapia con rHuEPO e dopo la sua sospensione temporanea. Al momento del secondo IVGTT, tre mesi dopo l'inizio della terapia, l'Hb, l'Ht e la pEPO erano significativamente più alti rispetto alla stimolazione basale. C'era un leggero aumento dei valori del

\begin{tabular}{|c|c|c|c|c|c|}
\hline & K-G & $\begin{array}{c}\text { Area IRI } \\
\mathrm{mU} / \mathbf{m l} \times \mathbf{m i n}\end{array}$ & $\begin{array}{c}\text { Area C-p } \\
\text { ng/ml x min }\end{array}$ & IGI & RI \\
\hline Prima & $1.47 \pm 0.15$ & $1.92 \pm 0.33$ & $217 \pm 30$ & $0.46 \pm 0.07$ & $1.33 \pm 0.18$ \\
\hline Dopo & $1.45 \pm 0.17$ & $1.74 \pm 0.31$ & $194 \pm 31$ & $0.45 \pm 0.06$ & $1.29 \pm 0.21$ \\
\hline
\end{tabular}

TAB. II - PARAMETRI CLINICI E DI LABORATORIO E INDICI DIALITICI IN 12 PAZIENTI EMODIALIZZATI PRIMA E DOPO TRE MESI DI TERAPIA CON rHuEPO E DOPO LA SUA SOSPENSIONE

\begin{tabular}{lccc}
\hline & Basale & $\begin{array}{c}\text { Terapia con } \\
\text { rHuEPO }\end{array}$ & $\begin{array}{c}\text { Sospensione } \\
\text { rHuEPO }\end{array}$ \\
\hline Emoglobina g/dl & $6.7 \pm 0.3$ & $11.3 \pm 0.6^{\mathrm{b}}$ & $11.0 \pm 0.6$ \\
Ematocrito \% & $20.3 \pm 1.1$ & $34.2 \pm 1.9^{\mathrm{b}}$ & $33.5 \pm 1.7$ \\
Eritropoietina plasmatica $\mathrm{ng} / \mathrm{ml}$ & $8.9 \pm 2.8$ & $136.5 \pm 48.6^{\mathrm{b}}$ & $7.2 \pm 2.4^{\mathrm{c}}$ \\
Urea ematica $\mathrm{mg} / \mathrm{dl}$ & $41.0 \pm 4.0$ & $45.0 \pm 2.0$ & $50.0 \pm 4.0$ \\
Creatinina sierica $\mathrm{mg} / \mathrm{dl}$ & $6.7 \pm 0.3$ & $7.4 \pm 0.2$ & $7.7 \pm 0.4$ \\
Acido urico sierico $\mathrm{mg} / \mathrm{dl}$ & $4.89 \pm 0.47$ & $5.31 \pm 0.47$ & $5.68 \pm 0.48$ \\
Fosforo sierico $\mathrm{mg} / \mathrm{dl}$ & $3.60 \pm 0.24$ & $3.83 \pm 0.50$ & $3.97 \pm 0.55$ \\
Kt/V & $1.14 \pm 0.05$ & $1.11 \pm 0.05$ & $1.12 \pm 0.05$ \\
Protein catabolic rate $\mathrm{g} / \mathrm{kg} / \mathrm{day}$ & $0.98 \pm 0.05$ & $1.07 \pm 0.06$ & $1.07 \pm 0.05$ \\
Peso secco $\mathrm{kg}$ & $60.1 \pm 2.8$ & $60.5 \pm 2.8$ & $60.5 \pm 2.8$ \\
\hline
\end{tabular}

${ }^{b} p<0.01$ vs Basale; ${ }^{c} p<0.01$ vs Terapia (test di Student-Newmann-Keuls) 


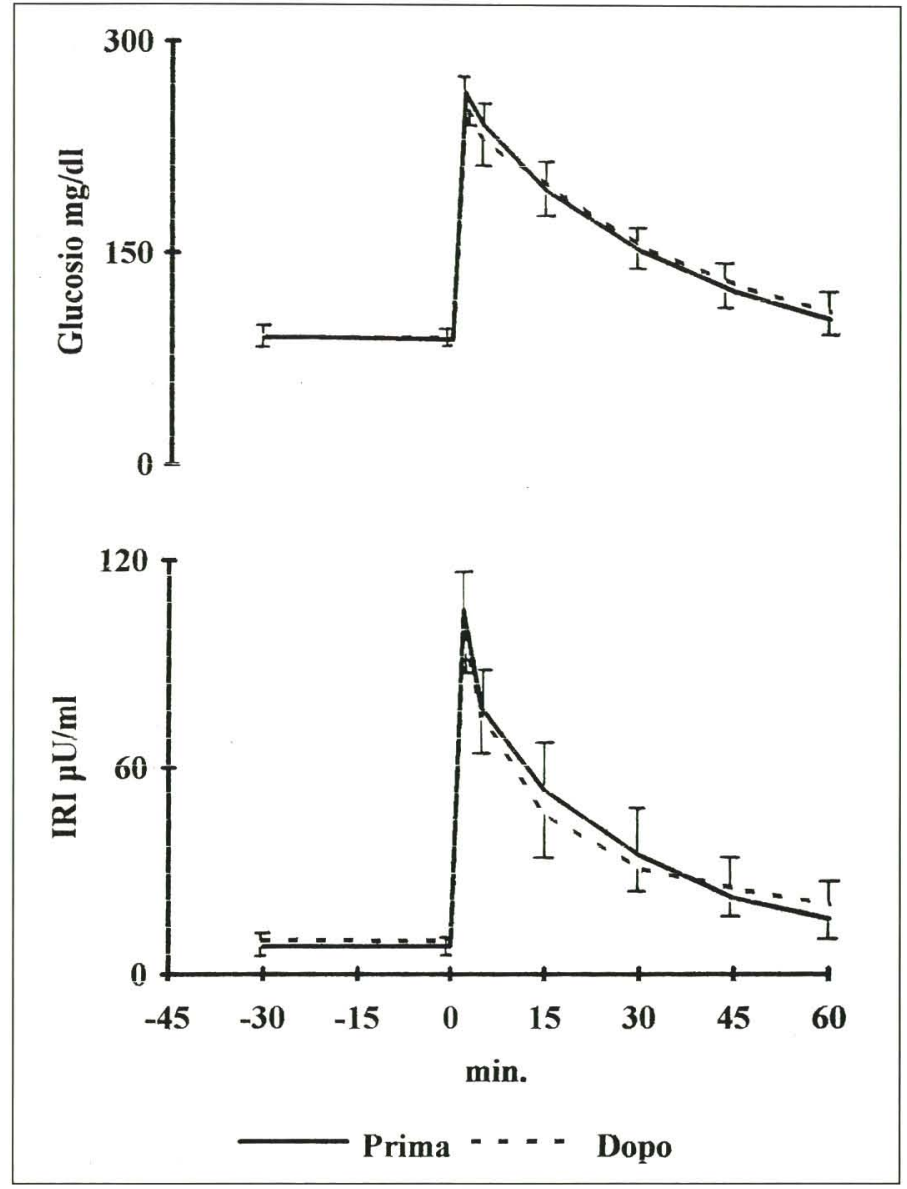

Fig. 1 - Valori plasmatici di glucosio e insulina durante IVGTT, prima e dopo somministrazione endovenosa di una singola dose di $\mathrm{rHuEPO}(50 \mathrm{UI} / \mathrm{kg}$ ) in 8 pazienti emodializzati.

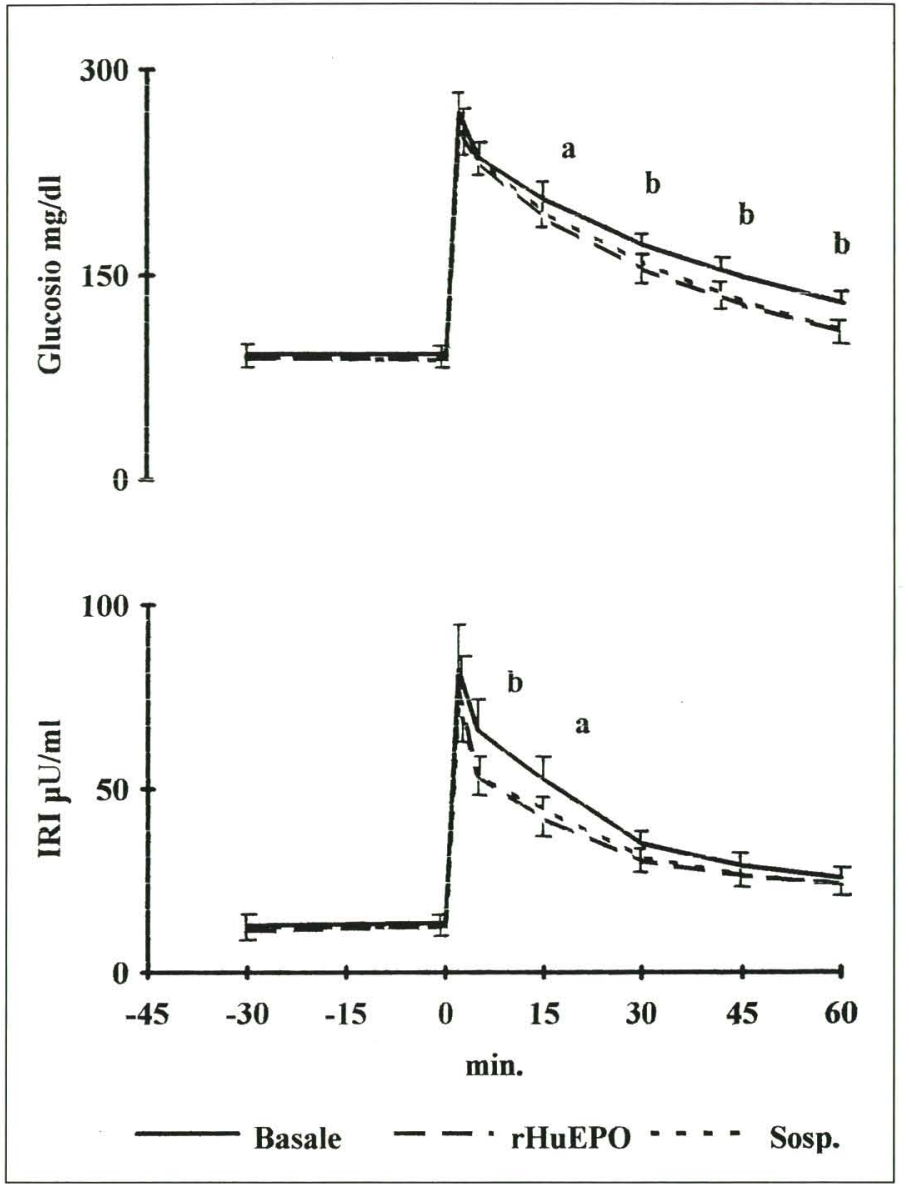

Fig. 2 - Valori plasmatici di glucosio e insulina durante IVGTT, prima e dopo 3 mesi di terapia con rHUEPO e dopo 2 settimane di sospensione temporanea della terapia, in 12 pazienti emodializzati.

$a=p<0.05 \quad b=p<0.01$ by Student-Newmann-Keuls test

TAB. III - PARAMETRI DEL METABOLISMO GLICIDICO, DERIVATI DALL'IVGTT, IN 12 PAZIENTI EMODIALIZZATI, PRIMA E DOPO TRE MESI DI TERAPIA CON rHuEPO E DOPO DUE SETTIMANE DI SOSPENSIONE TEMPORANEA DELL'rHuEPO

\begin{tabular}{lcccc}
\hline & K-G & $\begin{array}{c}\text { Area IRI } \\
(\mathbf{m} \text { U/ml)min }\end{array}$ & $\begin{array}{c}\text { Area C-p } \\
(\mathbf{n g} / \mathbf{m l}) \mathbf{m i n}\end{array}$ & IGI \\
\hline Basale & $1.07 \pm 0.10$ & $1.65 \pm 0.15$ & $245 \pm 35$ & $0.35 \pm 0.05$ \\
Terapia rHuEPO & $1.39 \pm 0.14^{\mathrm{b}}$ & $1.35 \pm 0.13^{\mathrm{b}}$ & $182 \pm 26^{\mathrm{a}}$ & $0.33 \pm 0.05$ \\
Sospens. $\mathrm{rHuEPO}$ & $1.36 \pm 0.14^{\mathrm{b}}$ & $1.41 \pm 0.16^{\mathrm{b}}$ & $201 \pm 24^{\mathrm{a}}$ & $0.33 \pm 0.06$ \\
\hline${ }^{a} p<0.05^{b} p<0.0$ vs Basale (test di Student-Newmann-Keuls) & & $1.00 \pm 0.07^{\mathrm{b}}$ \\
\hline
\end{tabular}

BUN, s-Cr, Pi e PCR, ma le differenze non raggiungevano la significatività statistica. Due settimane dopo la sospensione dell'rHuEPO, la pEPO è ritornata ai valori basali ma gli altri parametri non si sono modificati. I livelli plasmatici di G e IRI durante gli IVGTT sono mostrati in Figura 2 e i parametri del metabolismo glicidico, derivati dagli IVGTT, sono riassunti in Tabella III. Dopo tre mesi di terapia con $\mathrm{rHuEPO}$, i valori di $\mathrm{G}$ sono risultati significativamente più bassi a 15, 30, 45 e $60 \mathrm{~min}$, e i valori di IRI più bassi a 5 e $15 \mathrm{~min}$, ri- spetto alla stimolazione basale. Il K-G è aumentato significativamente, l'Area IRI, l'Area C-p e l'RI sono diminuiti significativamente, mentre i valori dell'IGI sono rimasti immodificati. Queste variazioni sono risultate pressoché uniformi in tutti i pazienti. Solo il caso 
che non ha riferito variazioni della sensazione generale di benessere e dell'attività fisica non ha mostrato variazioni del metabolismo glicidico. Nessuna correlazione è stata trovata tra modificazioni dell'Hb o dell'Ht e variazioni dei parametri del metabolismo glicidico. Due settimane dopo la sospensione dell'rHuEPO non si sono verificate ulteriori variazioni dei parametri del metabolismo glicidico.

\section{Discussione}

I nostri dati confermano un effetto positivo del trattamento a lungo termine con rHuEPO sul metabolismo glicidico nei pazienti emodializzati, principalmente legato ad una riduzione dell'insulinoresistenza. Dopo tre mesi di terapia con rHuEPO, tutti i pazienti, eccetto uno, hanno mostrato all'IVGTT una riduzione dei valori plasmatici di $\mathrm{G}$ e un miglioramento dei valori del K-G, insieme ad una riduzione delle aree IRI e di C-p e dei valori dell'RI. In contrasto con quanto riportato da altri Autori $(10,11,13)$, il nostro studio non ha rilevato alcun effetto dell'rHuEPO sulla secrezione insulinica. Kokot et al (10) in 5 MHP, esaminati con il test del pasto standard, hanno trovato un miglioramento dell'utilizzazione glicidica e della secrezione insulinica dopo terapia con rHuEPO e questi risultati sono stati confermati da Navino et al in 12 MHP (11). Al contrario Chagnac et al (13), in 10 MHP esaminati con oral glucose tolerance test, hanno trovato un peggioramento dell'utilizzazione glicidica e della secrezione insulinica dopo tre mesi di terapia con rHuEPO. Nella nostra esperienza la sensibilità al glucosio della ß-cellula pancreatica non sembra essere influenzata dalla terapia con rHuEPO, come dimostrato dalla mancanza di modificazioni dell'IGI. Il miglioramento dei valori del K-G può essere attribuito solo ad una migliore utilizzazione del glucosio da parte dei tessuti periferici, come dimostrato dalla riduzione dei valori di RI.

La diminuzione del rilascio di IRI e C-p, durante IVGTT, è la conseguenza fisiologica della minore richiesta insulinica da parte dei tessuti periferici. Questi risultati sono confermati da Borissova et al (12) che in 5 MHP diabetici, esaminati mediante euglycemic hyperinsulinemic clamp technique, hanno trovato un au- mento della sensibilità all'insulina del $50.7 \%$ dopo trattamento con rHuEPO. Il contrasto con i risultati di Kokot et al (10), Navino et al (11) e Chagnac et al (13) potrebbe dipendere dai differenti test utilizzati per studiare il metabolismo glicidico. I sopramenzionati Autori hanno condotto i loro esperimenti con il test del pasto standard $(10,11)$ o con l'oral glucose tolerance test (13) ed è noto che la secrezione insulinica da stimolazione orale è influenzata dalla percentuale di assorbimento del glucosio e dagli ormoni intestinali. È stato dimostrato che la terapia con rHuEPO può influenzare molti bersagli oltre alle cellule eritroidi (2429), per cui non può essere esclusa un'azione sulla funzione intestinale. L'IVGTT elimina le interferenze intestinali e può permettere una migliore valutazione della capacità secretiva della ß-cellula pancreatica.

Le modificazioni del metabolismo glicidico dopo terapia con rHuEPO non possono essere attribuite alla correzione dell'anemia. In questo studio non abbiamo trovato correlazioni tra modificazioni dell'Hb e modificazioni del K-G o dell'RI. È stato inoltre dimostrato che le anomalie del metabolismo glicidico non sono correlate al grado di anemia nei pazienti uremici dializati e non (16) e che la correzione dell'anemia mediante emotrasfusione non ha effetti sul metabolismo glicidico $(13,16)$. Anche un effetto diretto di alti livelli di pEPO su alcune tappe metaboliche può essere esclusa. Non ci sono state variazioni del metabolismo glicidico dopo somministrazione acuta di rHuEPO e dopo la sua sospensione, nonostante le signifilcative variazioni dei valori di pEPO.

Esiste la possibilità che l'aumento dell'attività fisica e dell'appetito, indotti dalla terapia con rHuEPO per alcuni mesi, possano aver migliorato lo stato nutrizionale e aumentato la massa muscolare con conseguente aumento del numero dei siti di utilizzazione del glucosio e riduzione dell'insulino resistenza. Un miglioramento dello stato nutrizionale nei MHP in terapia con rHuEPO è ampiamente riportato in letteratura (30-32). In questo studio noi non abbiamo trovato modificazioni del peso corporeo secco dopo tre mesi di terapia con rHuEPO ma è stato dimostrato che l'incremento del peso secco nel primo anno di trattamento con rHuEPO può essere mascherato dalla migliore disidratazione legata alla mi- gliore tolleranza dialitica (33). È interessante il fatto che solo il caso che non ha riferito variazioni della sensazione generale di benessere e dell'attività fisica non ha mostrato variazioni del metabolismo glicidico.

Alternativamente la terapia a lungo termine con rHuEPO potrebbe indurre un aumento della sintesi proteica e di conseguenza un miglioramento dell'attività di alcuni enzimi chiave della via glicolitica. Fischer et al (5) hanno riportato un miglioramento della percentuale di ritenzione proteica in $8 / 10$ MHP trattati con rHuEPO, e Riedel et al (6) una normalizzazione del pattern aminoacidico in 14 MHP dopo 12 mesi di terapia con rHuEPO. Inoltre il miglioramento dell'efficacia insulinica senza modificazioni del numero e dell'affinità dei recettori insulinici dopo terapia con rHuEPO riportato da Borissova et al (12), indica che l'aumento dell'utilizzazione glicidica è principalmente dovuto all'interferenza con un evento essenziale postrecettoriale insulino dipendente.

\section{Riassunto}

In 20 pazienti emodializzati (MHP) sono stati valutati, mediante intravenous glucose tolerance test (IVGTT), gli effetti dell'eritropoietina ricombinante umana sul metabolismo glicidico. In 8 casi gli IVGTT sono stati eseguiti prima e dopo una singola iniezione intravenosa di 50 $\mathrm{UI} / \mathrm{kg}$ di rHuEPO e in 12 casi prima e dopo tre mesi di terapia con $\mathrm{rHuEPO}$ alla dose di $50 \mathrm{UI} / \mathrm{kg} 3$ volte la settimana e dopo 2 settimane di sospensione dell'rHuEPO. Per ciascun test sono stati dosati glucosio, insulina (IRI) e C-peptide (C-p) e sono stati calcolati la costante di utilizzazione del glucosio (K-G), la produzione totale di IRI (Area IRI) e di C-p (Area C-p), l'indice insulinogenico (IGI), l'indice di insulino-resistenza (RI).

Dopo tre mesi di terapia con rHuEPO, il K-G è aumentato significativamente, l'Area IRI, l'Area C-p e l'RI si sono ridotti significativamente, mentre i valori dell'IGI sono rimasti pressocché invariati. Nessuna correlazione è stata trovata tra le modificazioni dei valori dell'Hb e quelle dei parametri del metabolismo glicidico. La somministrazione acuta di rHuEPO e la sua sospensione sono risultate prive di effetti sul metabolismo gli- 
cidico, nonostante le significative modificazioni dei valori plasmatici di eritropoietina.

La terapia prolungata con rHuEPO migliora significativamente il metabolismo glicidico nei MHP, principalmente per riduzione dell'insulino-resistenza. Né la correzione dell'anemia, né una azione diretta dell'rHuEPO su alcune tappe metaboliche sembrano essere responsabili di questi effetti.

\section{BIBLIOGRAFIA}

1. Scigalla P, Wieczorek L, Bicker U. Treatment of renal anemia with recombinant human erythropoietin: European experience. In: Garnick MB ed. Erythropoietin in Clinical applications. An international perspective. New York: Marcel Dekker 1990; 141-82.

2. Sobota JT. Erythropoietin treatment of end-stage renal disease: North American and Japanese experience. In: Garnick MB, ed. Erythropoietin in Clinical applications. An international perspective. New York: Marcel Dekker 1990; 183 219.

3. Winearls CG, Oliver DO, Pippard MJ, Reid C, Downing MR, Cotes PM. Effect of human erythropoietin derived from recombinant DNA on the anaemia of patients maintained by chronic haemodialysis. Lancet 1986; 2: 1175-8.

4. Eschbach JW, Egrie JC, Downing MR, et al. Correction of the anemia of end-stage renal disease with recombinant human erythropoietin. Results of a combined phase I and II clinical trial. N Engl J Med 1987; 316: 73-8.

5. Fischer Ch, Scigalla P, Park $\mathrm{W}$, et al. Influence of rHuEPO therapy on the protein metabolism of hemodialysis patients with terminal renal insufficiency. Contrib Nephrol 1989; 76: 250-6.

6. Riedel E, Hampl H, Scigalla P, Nundel M, Kessel M. Correction of amino acid metabolism by recombinant human erythropoietin therapy in hemodialysis patients. Kidney Int 1989; 36 (suppl 27): S216-21.

7. Acone D, Di Maio FS, Conte D, Giordano C. Effetto della terapia con rHuEPO sul metabolismo lipidico nel paziente in emodialisi. Giorn It Nefrol 1991; 8 (suppl 1): 469 (abstr.)

8. Mat O, Stolear JC, Georges B. Blood lipid profile in hemodialysis patients treated with human erythropoietin. Nephron 1992; 60: 236-7.

9. Viron B, Donsimoni R, Michel C, Al Khayat R, Mignon F. Effect of recombinant human erythropoietin on nutritional status and plasma lipids in uremic patients. Nephron 1992; 60: 249.

10. Kokot F, Wiecek A, Grzeszczak W, Klepacka J, Klin M, Lao M. Influence of erythropoietin treatment on endocrine abnormalities in hemodialyzed patients. Contrib Nephrol 1989; 76: 257-72.

11. Navino C, Barbè MC, Zamboni G, Chiarinotti D, Cusaro C, Verzetti G. La migliorata tolleranza glicidica dopo eritropoietina (EPO) negli emodializzati: azione diretta del farmaco o aumento dell'emoglobina? Atti del $32^{\circ}$ Congresso Nazionale della Società Italiana di Nefrologia, Bologna 1991. Bologna: Monduzzi 1991; 897-901.

12. Borissova AM, Djambazova A, Todorov K, Dakovska L, Tankova T, Kirilov G. Effect of erythropoietin on the metabolic state and peripheral insulin sensitivity in diabetic patients on hemodialysis. Nephrol Dial Transplant 1993; 8: 93.

13. Chagnac A, Korzets A, Zevin D, Levi J. Erythropoietin (EPO) impairs glucose tolerance in hemodialysis patients. Nephrol Dial Transplant 1992; 7: 766-7.

14. Guarnes V, Alvarez-Buylla R. Erythrocytes and glucose homeostasis in rats. Diabetes 1989; 38: 410-5.

15. Dionne KE, Colton CK, Yarmush ML. Effect of hypoxia on insulin secretion by isolated rat and canine islets of Langerhans. Diabetes $1993 ; 42$ : 12-21.

16. Allegra V, Mengozzi G, D’Achille F, Canciani D, Vasile A. Glucose-induced insulin secretion in uremia; role of anemia. Nephron 1992; 62: 246-7.

17. Allegra V, Mengozzi G, Vasile A. Iron deficiency in maintenance hemodialysis patients: assessment of 
diagnosis criteria and of three different iron treatments. Nephron 1991; 57: $175-82$.

18. Allegra V, Mengozzi G, Martimbianco L, Vasile A. Glucose-induced insulin secretion in uremia: effects of aminophylline infusion and glucose loads. Kidney Int 1990; 38: 1146-50.

19. Allegra V, Amendolagine F, Mengozzi G, Jesu L, Vasile A. Metabolic and hormonal assessment of patients on maintenance hemodialysis for 10 years or more and their importance in long-term survival. Nephron 1988; 49: 107-13.

20. Gotch FA, Sargent JA. A mechanistic analysis of the National Cooperative Dialysis Study (NCDS). Kidney Int 1985; 28: 52634.

21. Allegra V, Luisetto G, Mengozzi G, Martimbianco L, Vasile A. Glucose-induced insulin secretion in uremia: role of 1 alpha, $25(\mathrm{OH}) 2$-vitamin D3. Nephron 1994; 68: 41-7.

22. Allegra V, Mengozzi G, Martimbianco L, Vasile A. Glucose-induced insulin secretion in uremia: relation with acid-base balance and effects of bicarbonate administration. Nephron 1993; 63: 43-8.

23. de Fronzo RA, Ferranini E, Koivisto V. New concepts in the pathogenesis and treatment of noninsulin-dependent diabetes mellitus. Am J Med 1983; 17: 52-81.

24. Nissenson AR. Recombinant human erythropoietin: impact on brain and cognitive function, exercise tolerance, sexual potency, and quality of life. Semin Nephrol 1989; 9 (suppl 2): 25-31.

25. Suwata J, Maeda H, Ohmori N, Ohwa M, Ohtsuka H, Shimoyama H. Recombinant human erythropoietin therapy and autonomic nervous system. Nephron 1992; 61: 115-6.

26. Schaefer RM, Kokot F, Kuerner B, Zech M, Heidland A. Normalization of serum prolactin levels in hemodialysis patients on recombi- nant human erythropoietin. Int J Artif Organs 1989; 12: 445-9

27. Cases A, Escolar G, Reverter JC, et al. Recombinant human erythropoietin treatment improves platelet function in uremic patients. Kidney Int 1992; 42: 668-72.

28. Kimata H, Yoshida A, Ishioka C, et al. Human recombinant erythropoietin directly stimulates B cell immunoglobulin production and proliferation in serum-free medium. Clin Exp Immunol 1991; 85: 151-6.

29. Tsakiris D, Avramidis M, Karamitsos $\mathrm{K}$, et al. Cardiopulmunary function in haemodialysis patients before and after treatment of anaemia with erythropoietin. Kidney Int 1991; 39: 196.

30. Canaud B, Bouloux C, Rivory JP, et al. Erythropoietin-induced changes in protein nutrition: quantitative assessment by urea kinetic modeling analysis. Blood Purif 1990; 8: 301-8.

31. Barany P, Pettersson E, Ahlberg $\mathrm{M}$, et al. Nutritional assessment in anemic hemodialysis patients treated with recombinant human erythropoietin. Clin Nephrol 1991; 35: 270-9.

32. Toigo G, Situlin R, Vasile A, et al. Effects of erythropoietin administration on nutritional state and erythrocyte metabolism in maintenance hemodialysis patients. Contrib Nephrol 1992; 98: 79-88.

33. Kaupke CJ, Vaziri ND, Sampson JR, Atkins K. Effect of erythropoietin therapy on diet and dialysis clearances in hemodialysis patients. Int J Artif Organs 1990; 13: 218-22. 